\title{
Avaliação do potencial de uso da restrição hídrica em teste de sanidade de sementes de trigo visando à detecção de fungos ${ }^{1}$
}

\author{
Mauricio Mega Celano², José da Cruz Machado 3*, \\ David de Souza Jaccoud Filho ${ }^{4}$, Renato Mendes Guimarães ${ }^{5}$
}

\begin{abstract}
RESUMO - Objetivou-se, com o presente trabalho, avaliar a viabilidade do uso da restrição hídrica, em substituição ou alternativa ao uso da técnica de congelamento de sementes de trigo, no teste de incubação em substrato de papel, blotter test. $\mathrm{O}$ trabalho consistiu na comparação das duas técnicas por meio do exame de 60 amostras de sementes, coletadas em diferentes áreas do Estado de Minas Gerais e outras regiões do país, tendo como restritor hídrico o soluto manitol, no potencial de $-1,10 \mathrm{MPa}$, valor este determinado preliminarmente com base na capacidade de redução da germinação das sementes de trigo em condições do teste de sanidade. A incidência média de Bipolaris sorokiniana não foi afetada por nenhuma das técnicas comparadas, havendo, por outro lado, um maior estímulo da restrição hídrica em relação à Pyricularia grisea, Aspergillus ochraceus, Cladosporium cladosporioides e Epicoccum purpuracens. Para Alternaria alternata, Drechslera tritici-repentis e Fusarium graminearum, os maiores valores de incidência ocorreram no método do congelamento.
\end{abstract}

Termos para indexação: Triticum aestivum, patologia de sementes, blotter test.

\section{Potential of water restriction technique for wheat seed health testing}

\begin{abstract}
The objective of this study was to evaluate the use of the water restriction technique as an alternative to deep freezing for inhibiting wheat seed germination during blotter test incubation. The two techniques were compared using sixty seed samples collected in different areas of Minas Gerais state and other regions of Brazil. The water restriction established was determined by using manitol, at a potential of $-1.10 \mathrm{MPa}$, based on the results of preliminary essays. The mean incidence of $B$. sorokiniana was not affected by any of the two techniques. Values for the incidence of Pyricularia grisea, Aspergillus ochraceus, Cladosporium cladosporioides and Epicoccum purpuracens were higher when water restriction was used. On the other hand, values for the incidence of Alternaria alternata, Drechslera triticirepentis and Fusarium graminearum were lower with water restriction. Elongation of the seed radicle can be tolerated to a certain degree during blotter test incubation and the water restriction technique is a potential alternative of the freezing technique for detecting most of the fungi associated with wheat seeds.
\end{abstract}

Index terms: Triticum aestivum, seed pathology, blotter test.

\section{Introdução}

Inúmeras doenças que prejudicam a cultura do trigo podem ser disseminadas por sementes portadoras de seus

\footnotetext{
${ }^{1}$ Submetido em 11/04/2011. Aceito para publicação em 20/07/2012.

${ }^{2}$ Celano Rep. Com. Ltda, 84040-220, Ponta Grossa, PR, Brasil.

${ }^{3}$ Departamento de Fitopatologia, Universidade Federal de Lavras, 37200-000-Lavras, MG, Brasil.
}

agentes etiológicos. Além de danos diretos a cultura no campo, sementes portadoras de patógenos constituem focos primários para a cultura em fases posteriores de desenvolvimento. As plantas doentes produzem sementes

\footnotetext{
${ }^{4}$ Departamento de Fitossanidade, Universidade Estadual de Ponta Grossa, 84010-330-Ponta Grossa, PR, Brasil.

${ }^{5}$ Departamento de Agricultura, Universidade Federal de Lavras, 37200-000-Lavras, MG, Brasil.
}

\footnotetext{
*Autor para correspondência <machado@ufla.br>
} 
infectadas, que por sua vez garantem a sobrevivência das doenças por diferentes gerações (Reis et al., 2001).

No Brasil, dentre os patógenos transmitidos pelas sementes de trigo, destacam-se: Bipolaris sorokiniana (Sacc) Shoem. (agente etiológico da helmintosporiose ou mancha marrom e podridão comum das raízes), Drechslera tritici-repentis (Died) Shoem. (agente etiológico da mancha amarela), Gibberella zeae (Schw.) Petch (anamorfo: Fusarium graminearum Schwabe) (agente etiológico da giberela e podridão comum de raízes), Pyricularia grisea (Cooke) Sacc. (agente etiológico da brusone), Colletotrichum graminicola (Ces.) Wilson (agente etiológico da antracnose), Ustilago tritici Pers. (agente etiológico do carvão), Septoria tritici Rob. in Desm. (agente etiológico da mancha salpicada da folha) e Stagonospora nodorum (agente etiológico da septoriose, mancha da gluma e mancha do nó) (Reis et al., 2001).

A análise de sementes visando à avaliação da qualidade sanitária de uma amostra e, consequentemente, do lote que representa, é um pré-requisito fundamental do ponto de vista de defesa vegetal, certificação de sementes e de grande valor em programas de melhoramento, entre outros. A escolha do método utilizado na análise sanitária de sementes depende, entre vários fatores, do tipo de patógeno, das condições disponíveis e dos objetivos do teste (Machado, 1988, Machado et al., 2002).

A detecção de patógenos em sementes de trigo pode ser realizada por diversos métodos, sendo a incubação em substrato de papel (blotter test) e em meio agarizado os mais utilizados. Estes métodos são aplicados para quase todos os tipos de sementes, incluindo cereais, hortaliças, ornamentais e florestais (Neergaard, 1979).

Embora o método de incubação em substrato de papel, blotter test, com congelamento seja utilizado em todo o mundo com a finalidade de impedir a germinação das sementes de diversas espécies, principalmente das gramíneas, durante o período de incubação, ele apresenta algumas restrições. Trata-se de um teste que, por causar a morte das sementes, superestima a ocorrência de alguns fungos, incluindo os saprófitos, presentes nas sementes devido à eliminação do componente resistência do hospedeiro, além de constituir-se em obstáculo ao fluxo operacional de um maior número de amostras para análise em curtos períodos de tempo.

O uso da restrição hídrica em testes de sanidade de sementes para controlar o processo de germinação de sementes durante o período de incubação em substituição a2,4-Diclorofenoxiacetato de sódio ou uso do congelamento tem sido bem sucedido para alguns patossistemas, conforme relatados na literatura (Falleiro et. al., 2010, Carvalho et al., 2009, Machado et al., 2008; Machado et al., 2007, Machado et al., 2004; Coutinho et al., 2001).

Diante das dificuldades identificadas com o uso do método de congelamento para o teste de sanidade de sementes de trigo, o propósito deste trabalho foi avaliar a possibilidade de utilizar a técnica da restrição hídrica como alternativa à referida técnica, partindo-se do princípio que ela já tem sido empregada com vantagens em outros casos como arroz, sorgo, milho, cenoura para a mesma finalidade.

\section{Material e Métodos}

O trabalho foi conduzido no Laboratório de Patologia de Sementes, Departamento de Fitopatologia da Universidade Federal de Lavras (DFP-UFLA).

Origem das amostras de sementes de trigo: foram obtidas 60 amostras de sementes de trigo provenientes dos estados de Minas Gerais (1), Mato Grosso do Sul (2), Paraná (23), Rio Grande do Sul (7), Santa Catarina (7) e São Paulo (20).

Incubação das sementes em substrato de papel com congelamento: após a homogeneização das amostras, as sementes foram distribuídas em placas de Petri de $15 \mathrm{~cm}$ de diâmetro (25 sementes/placa de Petri), contendo três folhas de papel de filtro previamente esterilizadas e umedecidas em água destilada e esterilizada (2,5 vezes o peso do papel de filtro seco). As sementes, nas placas de Petri, foram inicialmente mantidas por 24 horas à temperatura de $20^{\circ} \mathrm{C} \pm 1{ }^{\circ} \mathrm{C}$, depois em congelador a $-20{ }^{\circ} \mathrm{C}$ por 24 horas e, posteriormente, incubadas a $20^{\circ} \mathrm{C} \pm 1{ }^{\circ} \mathrm{C}$ em câmara com fotoperíodo de 12 horas de luz negra (comprimento de onda próximo a ultravioleta), durante cinco dias.

Incubação das sementes em substrato de papel com restrição hídrica: para este teste, foram distribuídas sementes de trigo em placas de Petri de $15 \mathrm{~cm}$ de diâmetro (25 sementes/placa de Petri), contendo três folhas de papel de filtro previamente esterilizadas e umedecidas com solução osmótica (2,5 vezes o peso do papel de filtro seco) de manitol, potencial de $-1,1 \mathrm{MPa}(82,19 \mathrm{~g} / \mathrm{L})$, previamente esterilizadas. Para calcular a quantidade de manitol utilizada na preparação da solução, foi utilizado o programa SPMM (Michel e Radcliffe, 1995). A temperatura utilizada no cálculo foi de $20^{\circ} \mathrm{C}$, por ser esta a temperatura da câmara de incubação. As sementes foram incubadas em câmara com temperatura de $20{ }^{\circ} \mathrm{C} \pm 1{ }^{\circ} \mathrm{C}$ e fotoperíodo de 12 horas de luz negra (comprimento de onda próximo a ultravioleta) durante sete dias. 
Avaliação da incidência e intensidade dos fungos nas amostras de sementes: decorrido o período de incubação, procedeu-se ao exame individual das sementes ou plântulas, com auxílio de um microscópio estereoscópico, computando-se o percentual de incidência e intensidade de inóculo. Em alguns casos, a identificação foi confirmada pela visualização das estruturas morfológicas dos fungos em microscópio óptico. A intensidade de inoculo baseou-se no percentual da superfície de cada semente recoberta por estruturas típicas de cada espécie de fungo, individualmente. A escala visual de colonização dos fungos nas sementes foi constituída por quatro notas obedecendo às proporções: zero (nenhuma estrutura típica do fungo sobre as sementes); 1 (estrutura do fungo cobrindo até $10 \%$ do total da superfície das sementes); 2 (colonização entre 11 e $50 \%$ da superfície das sementes); 3 (colonização acima de $50 \%$ da superfície das sementes ).

Delineamento estatístico: o delineamento experimental utilizado foi o inteiramente casualizado (DIC), com dois tratamentos e oito repetições na avaliação do efeito dos métodos de incubação de sementes em substrato de papel com congelamento e com restrição hídrica na detecção de fungos nas sementes em teste. Cada parcela experimental foi constituída por uma placa de Petri, com 25 sementes.
Os dados relativos à incidência e intensidade de inóculo foram comparados pelo Teste $\mathrm{F}$.

\section{Resultados e Discussão}

Através da aplicação dos métodos de incubação de sementes em substrato de papel com congelamento e em substrato de papel com restrição hídrica foram detectados neste trabalho, em níveis variáveis, nas amostras de sementes examinadas, os fungos Bipolaris sorokiniana, Drechslera tritici-repentis, Fusarium graminearum, Pyricularia grisea, Penicillium spp., Aspergillus ochraceus, Alternaria alternata, Cladosporium cladosporioides e Epicoccum purpuracens (Figura 1A). Bipolaris sorokiniana e $F$. graminearum foram os fungos patogênicos de maior ocorrência, sendo detectados em $93,3 \%$ e $65,0 \%$ das amostras, respectivamente. As ocorrências generalizadas dos fungos A. alternata, $C$. cladosporioides e E. purpuracens, detectados em 60, 56 e 52 lotes, respectivamente (Figura 1A) devem-se, provavelmente, ao fato de que as sementes submetidas aos testes não sofreram desinfestação superficial. Estas espécies são consideradas contaminantes comuns em associação com sementes das mais diversas espécies de vegetais.
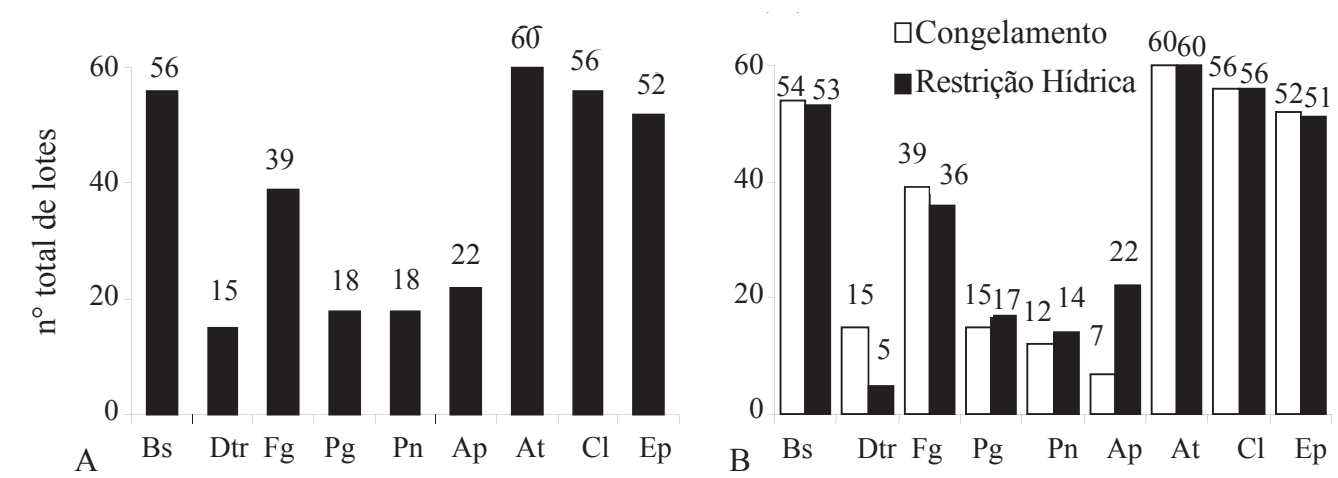

Figura 1. Número total de lotes (A) para cada fungo detectado em sementes de trigo submetidas ao teste de sanidade pelo método de incubação em substrato de papel com congelamento e substrato de papel com restrição hídrica (B) (total de 60 lotes). $\mathrm{Bs}=$ Bipolaris sorokiniana, $\mathrm{Dtr}=$ Drechslera tritici-repentis, $\mathrm{Fg}=$ Fusarium graminearum, $\mathrm{Pg}=$ Pyricularia grisea, $\mathrm{Pn}=$ Penicillium spp., Ap = Aspergillus ochraceus, $\mathrm{At}=$ Alternaria alternata, $\mathrm{Cl}=$ Cladosporium cladosporioides $\mathrm{e}$ $\mathrm{Ep}=$ Epicoccum purpuracens .

Pela análise comparativa realizada entre os dois métodos de detecção, D. tritici-repentis ocorreu em apenas cinco amostras pelo método de restrição hídrica, ao passo que pelo método de congelamento ele foi detectado em 15 amostras (Figura 1B). De um total de 39 lotes onde F. graminearum estava presente, pela técnica da restrição hídrica este fungo foi detectado em 3 desses lotes, sendo a incidência nestes casos de 3,5, 1,0 e 0,5\% (Figura 1B). Por sua vez, Aspergillus ochraceus, considerado um fungo de armazenamento, foi favorecido nas condições do método osmótico, estando presente em 22 lotes. Pelo método do congelamento este organismo ocorreu em apenas sete lotes (Figura 1B). 
Os valores médios de incidência dos fungos $D$. triticirepentis, $F$. graminearum e $A$. alternata pelo método do congelamento foram estatisticamente superiores do que aqueles registrados pelo método da restrição hídrica (Figura 2). Uma das hipóteses que pode explicar esse fato é o efeito de contaminação secundária entre sementes decorrente da elevada exsudação do conteúdo das sementes no substrato de papel, determinado pela ação do congelamento, que leva ao rompimento de estrutura celular e assim, proporcionando condições para o maior crescimento dos fungos nestas circunstâncias. Nestas condições verifica-se que a incidência de alguns fungos no lote é superestimada pelo método do congelamento.
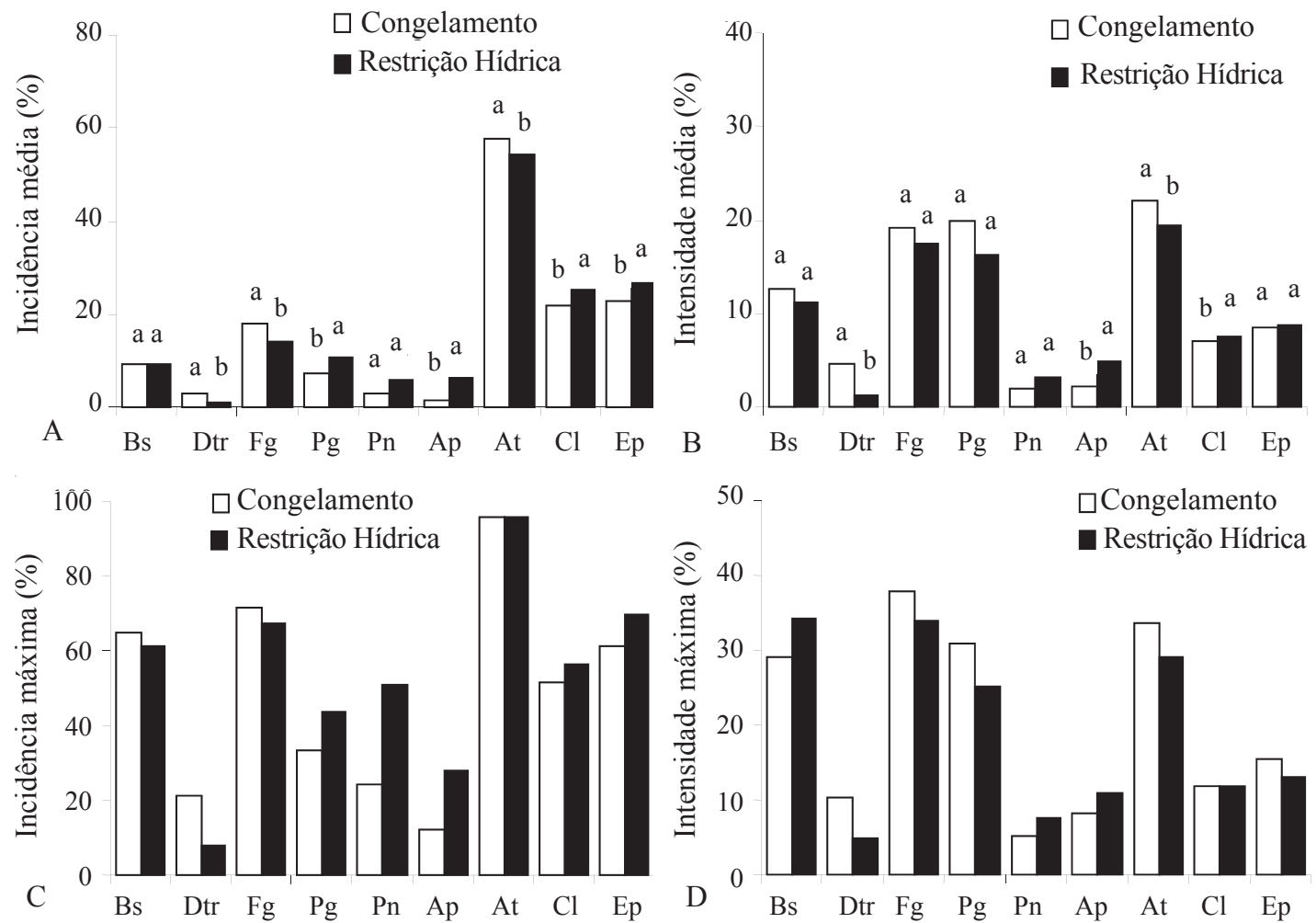

Figura 2. Valores médios (A e B) e máximos (C e D) de incidência e intensidade para cada fungo detectado em sementes de trigo submetidas ao teste de sanidade pelo método de incubação em substrato de papel com congelamento e substrato de papel com restrição hídrica (total de 60 lotes). Bs $=$ Bipolaris sorokiniana, Dtr $=$ Drechslera tritici-repentis, Fg = Fusarium graminearum, $\mathrm{Pg}=$ Pyricularia grisea, $\mathrm{Pn}=$ Penicillium spp., Ap = Aspergillus ochraceus, $\mathrm{At}=$ Alternaria alternata, $\mathrm{Cl}=$ Cladosporium cladosporioides e Ep $=$ Epicoccum purpuracens .

Outra hipótese levantada diz respeito à quebra de resistência da semente pela sua morte determinada pela ação do congelamento $\left(-18^{\circ} \mathrm{C} / 24\right.$ horas $)$. Com a resposta de defesa da semente reduzida, o desenvolvimento do fungo é facilitado. Em relação ao efeito da restrição hídrica, ela apenas inibe ou reduz a protrusão radicular, sem que ocorra morte da semente. Desta maneira, estando a semente fisiologicamente ativa, o crescimento do (s) fungo (s) contidos nessas sementes poderá sofrer a ação de certos metabólitos liberados pela semente. Portanto, os valores observados no método de incubação em substrato de papel com restrição hídrica parecem retratar com mais naturalidade o que ocorre nesta interação, ao contrário do que ocorre com o congelamento.

A incidência média dos fungos Pyricularia grisea, A. ochraceus, C. cladosporioides e E. purpuracens, pelo método da restrição hídrica, foi estatisticamente superior (Teste $\mathrm{F}, \mathrm{p} \leq 0,01$ ) àquela obtida pelo método do congelamento (Figura 2A). Possivelmente esses fungos possuam uma maior habilidade em desenvolverem-se em ambiente com maior potencial hídrico (-1,1 MPa), e também, em utilizar o manitol como fonte adicional de carbono, favorecendo o seu crescimento.

Em relação aos valores médios de intensidade de inóculo, foram detectadas diferenças estatísticas entre os dois métodos de sanidade comparados (Teste $F, p £ 0,01$ ) para 
os fungos $D$. tritici-repentis, A. alternata, $A$. ochraceus e $C$. cladosporioides. $\mathrm{O}$ método do congelamento proporcionou valores mais elevados aos dois primeiros fungos, ao contrário de A. ochraceus e C. cladosporioides (Figura 2B).

Estes resultados evidenciam que o método de incubação em substrato de papel com restrição hídrica, comparado ao método com congelamento, foi menos sensível na detecção de Drechslera tritici-repentis (Figuras 1 e 2). Os maiores valores de incidência deste organismo obtido a partir da avaliação das 60 amostras de sementes pelos métodos de congelamento e restrição hídrica foram de $21 \%$ e $8 \%$, respectivamente, sendo os valores médios de incidência nestas amostras de $3,1 \%$ e $0,9 \%$ para o método do congelamento e restrição hídrica. $D$. tritici-repentis produziu menos conidióforos e conídios nas sementes submetidas ao teste com restrição hídrica, por conseguinte sua detecção e quantificação foram dificultadas.

O uso de solução osmótica em testes de sanidade, especificamente no blotter test foi relatado inicialmente por Brodal (1997) na Noruega e Suécia com a finalidade de detectar espécies de Drechslera e Bipolaris em sementes de alguns cereais, de maneira indireta. Com a incorporação de uma solução de sacarose $0,5 \mathrm{M}$ ao substrato de papel, observou-se que sementes portadoras daqueles gêneros produziam logo abaixo ou ao seu redor uma coloração azulada. Segundo relatos de Sivanesan (1987), a formação e liberação de antraquinonas por aqueles gêneros eram os responsáveis por esta mudança de coloração no substrato. Em estudos comparativos entre o método de congelamento e o blotter osmótico, com base na análise de 57 amostras de sementes de cevada, no período de 1990-93, Brodal (1997) relata que o método osmótico (sacarose 0,5 M) proporcionou valores mais elevados de detecção daqueles fungos do que o blotter com congelamento. Relata ainda Brodal (1997) que além da maior sensibilidade do método osmótico, da ordem de $40 \%$, ele apresenta a vantagem de retardar a germinação das sementes. Neste trabalho o objetivo não era, portanto, determinar as espécies dos fungos em foco, mas sim a mudança de coloração do substrato em nível de gênero.

No presente trabalho, pelo método com restrição hídrica foi observada produção de uma coloração rosaclaro no substrato de papel na região abaixo das sementes que pertenciam a lotes em que, por meio do método do congelamento, foi detectado Drechslera tritici-repentis. Este fato faz com que a avaliação da incidência desse fungo possa ser realizada, pelo blotter com restrição hídrica, com base na formação daquela coloração. Esta constatação merece, no entanto investigações adicionais mais aprofundadas.

Este estudo deixa claro que o método de incubação em substrato de papel com congelamento é mais sensível que o método osmótico na deteç̧ão de fungos em programas voltados para a diagnose de patógenos de natureza quarentenária. Do ponto de vista de certificação, o método da restrição hídrica configura-se como uma alternativa das mais promissoras, sendo ele um método não destrutivo e que não oferece riscos para a saúde humana e nem envolve contaminantes químicos em laboratórios onde sementes são manipuladas. É válido lembrar que, pelo método de restrição hídrica, o fluxo operacional de análise de amostras em laboratório de rotina não sofre restrições como no caso do uso do blotter test com congelamento. Além dessas vantagens, por meio do blotter com restrição hídrica, a manipulação das sementes em pré-incubação é menor, o que previne problemas resultantes da movimentação indesejável das sementes nos substratos do blotter test.

\section{Conclusões}

A técnica da restrição hídrica pode ser empregada com sucesso na análise de sementes de trigo, em programas de certificação, como alternativa à técnica do congelamento pelo teste de Incubação em Substrato de Papel- blotter test, para a detecção de parte dos fungos fitopatogênicos e de deterioração em armazenamento associados a estas sementes.

A recomendação da restrição hídrica na detecção de alguns dos fungos como Drechslera tritici-repentis e Fusarium graminearum ainda requer estudos adicionais.

\section{Agradecimentos}

Ao Eng ${ }^{\circ}$. Agr. Amando Ribas Stabach. Ao Dr. Augusto Cesar Pereira Goulart. Ao Eng. Agr. Anderson Feltrin. Ao Engo. Agr. Carlos Utiamada. À Dra. Maria Heloisa D. Moraes e Dra. Norimar Denardin, pelo fornecimento de parte dos lotes de sementes. Ao CNPq/CAPES/FAPEMIG, pelo apoio financeiro e logístico na condução destes estudos.

\section{Referências}

BRODAL, G. Comparative tests with the osmotic blotter method for detection of Drechslera spp. in barley seeds. In: HUTCHINS, J.D.; REEVES, J.C. Seed health testing: progress towards the 21st century. NIAB / ISTA / CAB International, 1997. p.211-218. 
CARVALHO, E.M.; SILVA, U.A.; RODRIGUES, D.C.G.A. Uso da restrição hídrica na detecção de Alternaria dauci e Alternaria radicina em sementes de cenoura (Daucus carota). Tropical Plant Pathology, v.34, p.216222, 2009. http://www.scielo.br/scielo.php?script=sci_arttext\&pid=s1982$56762009000400003 \& \operatorname{lng}=$ en\&nrm=iso

COUTINHO, W.M.; MACHADO. J.C.; VIEIRA, M.G.G.C.; GUIMARÃES, R.M.; FERREIRA, D.F. Uso da restrição hídrica na inibição ou retardamento da germinação de sementes de arroz e feijão submetidas ao teste de sanidade em meio ágar-água. Revista Brasileira de Sementes, v.23, n.2, p.127-135, 2001. http://www.abrates.org.br/portal/revista/_apps/revista/artigospublicados/ indice.php? $\mathrm{v}=23 \& \mathrm{n}=2$

FALLEIRO, B.A.S.; ALMEIDA, P.B.A.; COUTINHO, W.M.; SUASSUNA, N.D.; KOBAYASTI, L. Use of osmotic solutions for inhibition of sunflower seed germination in blotter test. Tropical Plant Pathology, v.35, v.6, 2010. http://www.scielo.br/scielo.php?script=sci_arttext\&pid=S1982$56762010000600002 \& \operatorname{lng}=\mathrm{en} \& \mathrm{nrm}=\mathrm{iso}$

MACHADO, J.C. Patologia de sementes: fundamentos e aplicações. Brasília. MEC/ESAL/FAEPE. 1988. 107p.

MACHADO, J.C.; LANGERAK,C.C.J.; FILHO JACCOUD, D.S. (Ed.). Seed-borne fungi: a contribution to routine seed health analysis. Zurich: ISTA, 2002. 138p.

MACHADO, J.C.; GUIMARÃES, R.M.; VIEIRA, M.G.G.C.; SOUZA, R.M.; POZZA, E.A. 2004. Use of water restriction technique in seed pathology. Seed Testing International, v.128, p.14-28 (ISTA News Bulletin n.128, 2004).
MACHADO, J.C.; COUTINHO, W.M.; GUIMARÃES, R.M.; VIEIRA, M.G.G.C.; FERREIRA, D.F. Use of osmotic solutes to control seed germination of rice and common bean in seed health blotter tests. Seed Science and Technology, v.36, p.66-75, 2008. http://www.ingentaconnect. $\mathrm{com} /$ content/ista/sst/2008/00000036/00000001/art00007;jsessionid $=4 \mathrm{c} 1$ u46doq8dka.alice

MACHADO, A.Q.; MACHADO, J.C.; VIEIRA, M.G.G.C.; CASSETARI NETO, D.; SOUZA, M.V. Potencial do uso da restrição hídrica em testes de sanidade de sementes de algodoeiro. Fitopatologia Brasileira, v.32, n.5, 2007. http://www.scielo.br/pdf/fb/v32n5/v32n5a06.pdf

MICHEL, B.E.; RADCLIFFE, D.A. Computer program relating solute potential to solution composition for five solutes. Agronomy Journal, v.87, p.126-130, 1995.

NEERGAARD, P. Seed pathology. 2.ed. London: MacMillan, 1979. 2v. $1191 \mathrm{p}$.

REIS, E.M.; CASA, R.T.; MEDEIROS, C.A. Diagnose, patometria e controle de doenças de cereais de inverno. Londrina: E.S. Comunicação S/C Ltda, 2001, 94p.

SIVANESAN, A. Graminicolous species of Bipolaris, Curvularia, Drechslera, Exserohilum and their teleomorphs. C.A.B. International Mycological Institute, 1987. 261p. (Mycological Papers, 158). 